\title{
Multisensory Exercise Improves Balance in People with Balance Disorders: A Systematic Review*
}

\author{
Su-lin $\mathrm{ZHANG}^{1,2 \dagger}$, Dan $\mathrm{LIU}^{1 \dagger}$, Dong-zhen $\mathrm{YU}^{3 \dagger}$, Yu-ting ZHU${ }^{1}$,Wen-chao XU1 ${ }^{1}$, E TIAN ${ }^{1}$, Zhao-qi GUO ${ }^{1}$, Hai-bo SHI ${ }^{3}$, \\ Shan-kai YIN ${ }^{3 \#}$, Wei-jia KONG ${ }^{1,2 \#}$ \\ ${ }^{I}$ Department of Otorhinolaryngology, Union Hospital, Tongji Medical College, Huazhong University of Science and Technology, \\ Wuhan 430022, China \\ ${ }^{2}$ Institute of Otorhinolaryngology, Union Hospital, Tongji Medical College, Huazhong University of Science and Technology, \\ Wuhan 430022, China \\ ${ }^{3}$ Department of Otorhinolaryngology Head and Neck Surgery, Shanghai Jiao Tong University Affiliated Sixth People's Hospital, \\ Shanghai 200233, China
}

(C) The Author(s) 2021

\begin{abstract}
[Abstract] Objective: To examine the effect of multisensory exercise on balance disorders. Methods: PubMed, Scopus and Web of Science were searched to identify eligible studies published before January 1, 2020. Eligible studies included randomized control trials (RCTs), non-randomized studies, case-control studies, and cohort studies. The methodological quality of the included studies was evaluated using JBI Critical Appraisal Checklists for RCTs and for Quasi-Experimental Studies by two researchers independently. A narrative synthesis of intervention characteristics and healthrelated outcomes was performed. Results: A total of 11 non-randomized studies and 9 RCTs were eligible, including 667 participants. The results supported our assumption that multisensory exercise improved balance in people with balance disorders. All of the 20 studies were believed to be of high or moderate quality. Conclusion: Our study confirmed that multisensory exercise was effective in improving balance in people with balance disorders. Multisensory exercises could lower the risk of fall and enhance confidence level to improve the quality of life. Further research is needed to investigate the optimal strategy of multisensory exercises and explore the underlying neural and molecular mechanisms of balance improvement brought by multisensory exercises.
\end{abstract}

Key words: multisensory exercises; multisensory integration; balance; systematic review

Balance maintenance is essential for daily life. Balance can be defined as a stable state achieved by maintaining the body's mass center within manageable limits of stability ${ }^{[1,2]}$. Static balance is attained by keeping the center of mass within the base of support during standing and sitting ${ }^{[2-4]}$. On the other hand, dynamic balance is more challenging because it requires maintenance of equilibrium of the body when both the center of mass and base of support are not constant $^{[2,5]}$. Ability to maintain balance is the basis of

Su-lin ZHANG, E-mail: 156933024@qq.com; Dan LIU, E-mail: 469783007@qq.com; Dong-zhen YU, E-mail: drdzyu @ $126 . c o m$

†These authors contributed equally to this work.

\#Corresponding authors, Wei-jiaKONG, E-mail: entwjkong@, hust.edu.cn; Shan-kai YIN, E-mail: yinshankai@china.com

*This work was supported by grants from the National Twelfth Five- Year Research Program of China (No. 2012BAI12B02), the National Natural Science Foundation of China (No. 81873701), and the Two-hundred Talent Fund of Medical College of Shanghai Jiao Tong University (No. 20191921). movement and performance of daily activities. Balance disorder refers to impaired or lost ability to accomplish equilibrium. The causes of imbalance include visual loss such as glaucoma, vestibular failure such as Meniere disease, somatosensory loss such as diabetic peripheral neuropathy, cerebellar lesions such as cerebellar stroke, and lesions involving basal ganglia and frontal lobes, etc. Balance disorders fall into three categories in terms of sensorimotor levels ${ }^{[6]}$. The lowest-level sensorimotor dysfunction involves musculoskeletal dysfunction or multisensory disturbances such as visual, vestibular and somatosensory disturbances ${ }^{[7]}$. The middle-level and the highest-level sensorimotor dysfunction separately involves faulty execution and selection of postural and locomotor responses ${ }^{[7]}$. Middle-level dysfunction includes spastic, ataxic, dystonic, and choreic gaits, for instance, cerebellar $\operatorname{ataxia}^{[7]}$. The highest-level balance disorders include subcortical disequilibrium, frontal disequilibrium and so on, e.g. Parkinson's disease ${ }^{[7]}$. Moreover, balance disorder also increases the risk of fall ${ }^{[8,9]}$ and even leads to fall-related injuries. According to WHO, roughly 
646000 suffered from fatal falls each year, and the fall represents the second leading cause of unintentional injury-related death $(2018)^{[10]}$. Among people over 60 years, the rate of fall-related death is the highest around the globe ${ }^{[10]}$. Though not fatal, approximately 37.3 million falls that require medical attention take place each year ${ }^{[10]}$. To ameliorate balance disorders and reduce possible injuries, clinicians should understand the mechanism of balance disorders and thereby work out effective management.

Medication and surgery are seldom used for balance improvement and fall prevention. The main management for improving balance includes physical exercises, vestibular rehabilitation and multisensory exercises. They are three different training programs that are used to manage balance disorder ${ }^{[11-13]}$.

Physical exercises include aerobic exercise, stretching exercise and resistance training, among others $^{[11,14,15]}$. Those exercises train people's muscles, stretch their bodies, and challenge the ability to respond to different situations. Many studies have shown that physical exercises could bring beneficial effects on balance ${ }^{[11,14-16]}$. Vestibular rehabilitation includes adaptation, habituation, sensory substitution and optokinetic exercises ${ }^{[17]}$. Vestibular rehabilitation aims to facilitate the compensation for vestibular function ${ }^{[17]}$. It has been shown to be an effective treatment for reducing vertigo and improving gaze stabilization and balance ${ }^{[12,18]}$. Multisensory exercises consist of activities simultaneously stimulating at least two sensory modalities of multiple sensory systems including visual, auditory, tactile, vestibular, somatosensory systems and so on ${ }^{[13,19,20]}$. Multisensory exercises may improve balance by enhancing central nervous system (CNS) ability to process and integrate sensory afferents and facilitating compensation for deficient sensory inputs ${ }^{[20,21]}$. And this process may rely on improved connectivity between numerous interconnected neural circuits that are regulated by the CNS.

Multisensory exercises and vestibular rehabilitation both aim to promote balance by modulating CNS plasticity ${ }^{[22,23]}$, while physical exercises aim at enhancing physical function ${ }^{[16]}$. From the perspective of sensory inputs, multisensoryexercisesstimulatemultiplesensory systems simultaneously ${ }^{[13]}$, but vestibular rehabilitation mainly stimulates the unisensory system such as vision or somatosensory sensation ${ }^{[17]}$. Multisensory exercises can facilitate CNS compensation for multisensory afferents, vestibular rehabilitation is mainly aimed at patients with vestibular dysfunction to promote their vestibular compensation ${ }^{[17]}$. The comparisons among three training programs are shown in table 1 .

While a few studies have reported that multisensory exercises could positively impact on balance disorder ${ }^{[24,25]}$, the effects of multisensory exercises on balance have not been systemically investigated. In this study, we conducted a systematic review with an attempt to look into the effect of multisensory exercise on balance disorder.

\section{MATERIALS AND METHODS}

\subsection{Study Design and PICO}

Patients with balance disorders under multisensory exercises were included in this systematic review. Comparators were patients with balance disorders undergoing other exercises or no multisensory training. In this study, balance function was the main outcome indicator.

\subsection{Protocol of Systematic Review}

The protocol for this systematic review was registered in PROSPERO (registration number: CRD42020151453). Protocol details were available from https://www.crd.york.ac.uk/prospero/display_ record.php?ID=CRD42020151453.

\subsection{Search Strategy and Study Selection}

This systematic review was performed in accordance with the PRISMA statement ${ }^{[26]}$. Two reviewers independently conducted the literature search. Electronic databases, including PubMed, Scopus and Web of Science, were searched to identify

Table 1 Comparison among physical exercises, vestibular rehabilitation and multisensory exercises

\begin{tabular}{llll}
\hline & \multicolumn{1}{c}{ Physical exercises } & \multicolumn{1}{c}{ Vestibular rehabilitation } & \multicolumn{1}{c}{ Multisensory exercises } \\
\hline Target population & Various disorders & Vestibular dysfunction & Balance disorders \\
& (e.g. stroke and dementia) & (e.g. BPPV and Menière's disease) & (e.g. eye disorders and type 2 diabetes) \\
Principles & Physical function: & Unisensory stimulation: & Multisensory stimulation: \\
& Train muscles & Vision (vestibulo-ocular reflex), & Vision, hearing, touch, vestibular \\
& Stretch bodies & somatosensory and vestibular & and somatosensory sensation, among \\
& Respond to different situations & sensation & others \\
& & Habituation: & \\
& & Repeated exposure & \\
& & Sensory substitution: & \\
& Visual or somatosensory sensation & \\
& & By compensating for vestibular & By enhancing multisensory integration \\
How to improve balance & By enhancing physical & dysfunction & \\
\hline
\end{tabular}

BPPV: benign paroxysmal positional vertigo 
eligible studies published before January 1, 2020. We used the following search strategies: (1) (Multisensory OR Multi-sensory OR Multisensorial) OR (Crossmodal OR Cross-modal) OR (Crossmodal Sensory) OR (Multimodal OR Multimodality) OR Intersensory OR Multisystem), (2) (Exercis* OR Exercise) OR (Exercise Therapy) OR Train* OR Rehabilitat* OR Treat* OR Intervent* OR Program* OR Therap* OR Stimulation, and (3) Balanc* OR Equilibrium OR Gait OR Stability OR Stabilization OR Sway OR Instability OR Postur* OR (Gesture Control) OR (Movement Control). The "*" was the wild card. These search queries were combined by Boolean operator "And".

The articles published in English were included and duplicates were excluded. Two reviewers (SZ and DL) assessed all the articles against the inclusion and exclusion criteria by reading title, abstract and the fulltext. If any disagreement occurred, a third reviewer (DY) would be consulted, and a consensus was arrived at by referring to the inclusion and exclusion criteria.

The systematic review pooled the most up-todate multisensory exercise that improves balance in people with balance disorders. A study was included if: (1) Participants included children, adolescents, adults and elders diagnosed with diseases that might affect balance, those with fall history or complained instability or assessment showed impaired balance function; (2) Multisensory exercise was the main intervention; (3) At least one outcome measure involved balance; (4) Included RCTs, non-randomized studies, case-control studies, and cohort studies; (5) Sample size $>10$. Moreover, studies were excluded if: (1) They were reviews, case reports, conference abstracts, comments, or letters; (2) Data were unavailable or insufficient; (3) They had overlapping dates or were animal studies.

\subsection{Quality Assessment}

The JBI Critical Appraisal Checklists for RCTs and for Quasi-Experimental Studies were used to assess the methodological quality of the included papers $(n=20)$. The checklist for evaluating the RCTs consisted of 13 assessment criteria and the one for the quasi-experimental studies consisted of 9 assessment criteria. Each criterion was rated as "yes," "no," "unclear," or "not applicable."[27].

Two reviewers (SZ and DL) independently assessed the quality of eligible studies, which were rated as "include," "exclude," or "seek further information." In case of disagreement, a third reviewer (DY) was consulted to achieve a consensus. All disagreements with regard to the methodological quality of the studies were discussed and agreed upon by the team. The level of methodological quality was determined as follows: fair quality if less than $50 \%$ of the items were rated as "yes," moderate quality if $51 \%$ to $80 \%$ of the items were rated as "yes," and good quality if more than $80 \%$ of the items were rated as "yes." No studies were excluded based on methodological quality.

\subsection{Data Extraction}

The relevant data of the studies were extracted, including authors, publication year, country, design, sample population, sample size, age, intervention (frequency and duration) and outcome measures. Two reviewers (SZ and DL) separately extracted data which were input into a form. If any disagreement occurred, they reviewed the full text of the articles to reach an agreement.

\subsection{Data Analysis}

The authors intended a priori to complete a metaanalysis. However, the limited number of articles retrieved and substantial heterogeneity across the retrieved studies did not allow meta-analysis of the results for different study characteristics. In view of these, we only provided a qualitative synthesis of the results.

\section{RESULTS}

\subsection{Study Selection and Characteristics}

The electronic database search yielded 8460 records, of which 1726 were identified by Endnote as duplicates. Another 680 duplicate records were removed manually, leaving 6054 potentially relevant records for future screening. Upon reading the title and the abstract, we removed records that did not satisfy the inclusion criteria. Finally, 401 potentially eligible articles were subjected to full-text screening and 20 of them were eventually included in the systematic review (fig. 1). The study characteristics separately for

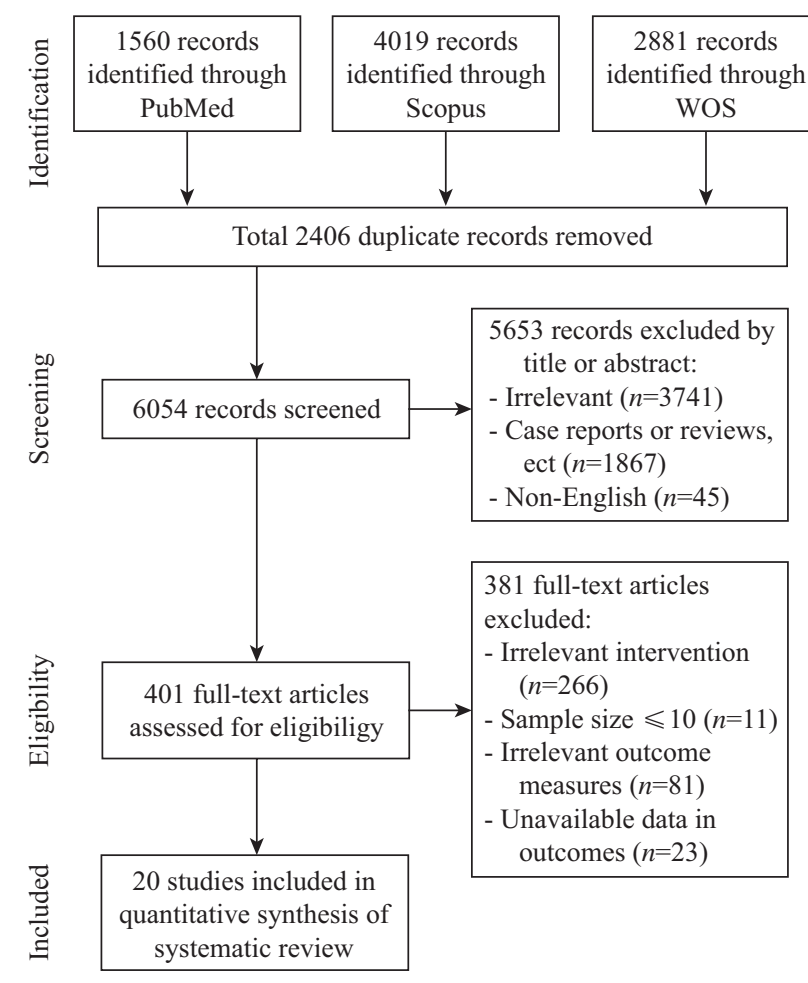

Fig. 1 Flow diagram of study selection process 
RCTs and nonRCTs, included mean age, sex, types of disorder/disease, and most importantly, the types of interventions, range of length of interventions, a dose of intervention (i.e. No. of sessions), length of each session, mode of delivery, main result, etc.

\subsection{Participants and Settings}

The studies included in this review contained a total of 667 participants ranging from $10^{[28]}$ to $68^{[29]}$ participants per study (table 2). The mean age of the study samples ranged from $133.5 \pm 26.9$ months ${ }^{[30]}$ to $84.8 \pm 4.1$ years ${ }^{[31]}$. Two studies collected and reported information about multisensory exercise, which included participants all females ${ }^{[31,32]}$. Of these participants, 30 were ataxic patients ${ }^{[33]}, 73$ were older adults with a history of falls ${ }^{[20,34,35]}, 33$ were older adults without fall history ${ }^{[35,36]}, 18$ were older adults with visual impairment ${ }^{[37]}, 16$ were diabetics with peripheral neuropathy ${ }^{[38]}$, and 21 were patients with essential tremor ${ }^{[39]}$. A total of 667 participants were included in the 20 studies, four studies were conducted in the United States, four in Canada, two in Brazil, and two in France, other studies in England, Indian, Sweden, and so on.

\subsection{Intervention Programs}

Table 2 presents the characteristics of the exercise training interventions. The total time spent in exercising during the multisensory exercise interventions varied from $0.5^{[30,40]}$ to $180 \mathrm{~h}^{[33]}$, while the intervention duration ranged from $2^{[41]}$ to 16 weeks ${ }^{[31]}$. Studies of intervention time showed that multisensory exercises worked within 6 weeks $^{[34,38,42]}, 8$ weeks ${ }^{[20,39,43]}, 12$ weeks $^{[36,37,44]}$ ) respectively. However, there are still studies which reported effective intervention duration was less than 6 weeks or more than 12 weeks. Six of the interventions were new systems or advanced equipment such as the Balance Freedom TM head-band system (Balance Int. Innovations $\mathrm{GmbH}$, Switzerland), a performance scoring system, etc. ${ }^{[28,39-41,43,45]}$. Two of the interventions used games for multisensory training ${ }^{[30,35]}$. One study used a multisensory program with dance ${ }^{[37]}$. One study involved cognitive training in addition to multisensory training ${ }^{[46]}$.

\subsection{Group Comparison}

One study ${ }^{[46]}$ used three groups: one control group (conventional training included balance tasks in seated, standing, and moving task situations), one group of individual Dual-Task Training, and one group of multisensory training. Five studies ${ }^{[28,33,34,39,42]}$ used only one group in their study design. The other studies used two groups (intervention group and control group). The main control groups included oral education, daily routine, conventional training, strength exercise, and other none-multisensory exercises (table 2).

\subsection{Outcomes Measures about Balance}

The measures used for balance assessment include the sensory organization test (SOT) $(n=3)$, the timed up and go test (TUG) $(n=4)$, the Berg balance scale (BBS) $(n=3)$ and the activities-specific balance confidence scale $(\mathrm{ABC})(n=4)$. This systematic analysis showed that multisensory exercises were effective in improving balance in people with balance disorders. The balance performance was assessed by the SOT, TUG, BBS and $\mathrm{ABC}$. The $\mathrm{SOT}^{[47]}$ examines the abilities to maintain standing balance under six different sensory conditions: (1) eyes are fixed on a fixed screen when standing on a fixed surface; (2) eyes close while standing on a fixed surface; (3) eyes are fixed on a moving screen when standing on a fixed surface; (4) eyes are fixed on a fixed screen while standing on moving surface; (5) eyes close when standing on a moving surface; (6) eyes are fixed on a moving screen when standing on a moving surface. The $\mathrm{TUG}^{[48]}$ measures the time taken to rise from an arm chair, walk 3 meters away, go back and sit down again. The $\mathrm{BBS}^{[49]}$ consists of 14 activities rated on a $0-4$ point scale, with the maximum score being 56 . The major activities are dynamic tasks ranging from simple items (e.g., sit-tostand) to difficult items (e.g., turning $360^{\circ}$ ). A recent study suggested that only an over 8-point change in BBS could lead to clinically significant functional alteration ${ }^{[50]}$. However, the change in BBS in our study was too small to produce a clinically significant change. Nonetheless, multisensory exercises pose no harm to balance performance in any way. The $\mathrm{ABC}^{[51]}$ is a 16item questionnaire that examines balance confidence. Items are rated from $0 \%$ (no confidence) to $100 \%$ (full confidence). The activities' difficulty ranges from walking around house to walking on icy sidewalks. Higher ABC score implies better balance confidence during daily activities ${ }^{[52]}$. Stronger self-assurance can break up the vicious circle between fear of fall and avoidance of physical activity.

\subsection{Methodological Quality of Included Studies}

The eligible studies are listed in table 2. The methodological quality assessment of 9 RCTs and 11 non-randomized studies are presented in tables 3 and 4 . Eight RCTs scored "yes" for most items. Two studies were unclear randomization used for assignment of participants to treatment groups and the allocation to treatment groups concealed unclear too ${ }^{[36,45]}$. In one study, participants blind to treatment assignment were unclear ${ }^{[40]}$. Only two studies answered yes for delivering treatment blind to treatment assignment ${ }^{[31,46]}$. Outcomes assessors in three studies were blind to treatment assignment by methodological quality assessment ${ }^{[31,40,46]}$. Our study had no follow-up assessment because of time constraints ${ }^{[46]}$. The randomization process was not clearly described in 11 non-randomized studies. Ten studies scored "yes" for most items, except for item $1^{[33]}$. There was no control group in the four studies ${ }^{[35,37,41,43]}$. Two studies' outcomes measured whether a reliable way were unclear ${ }^{[33,35]}$. 


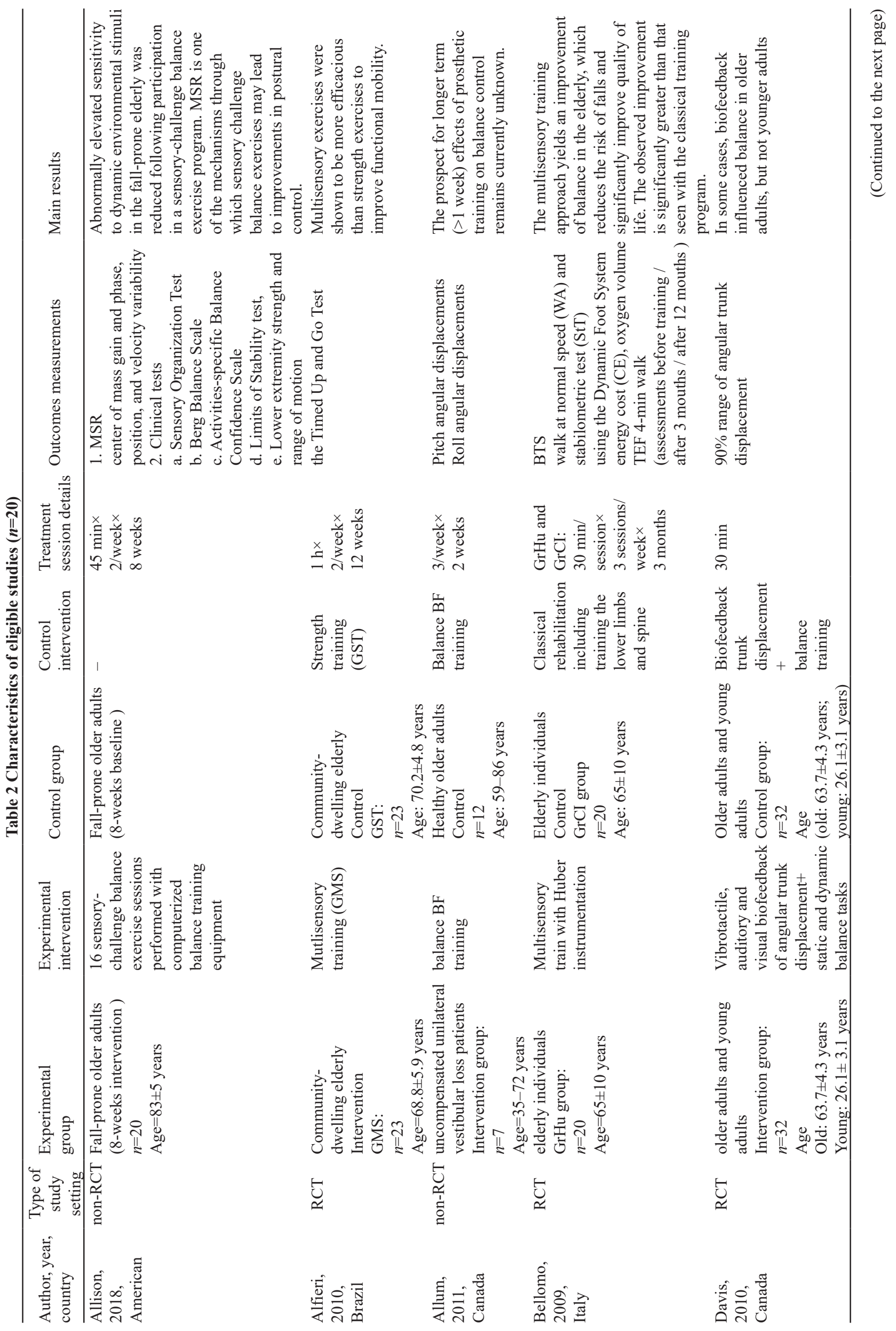




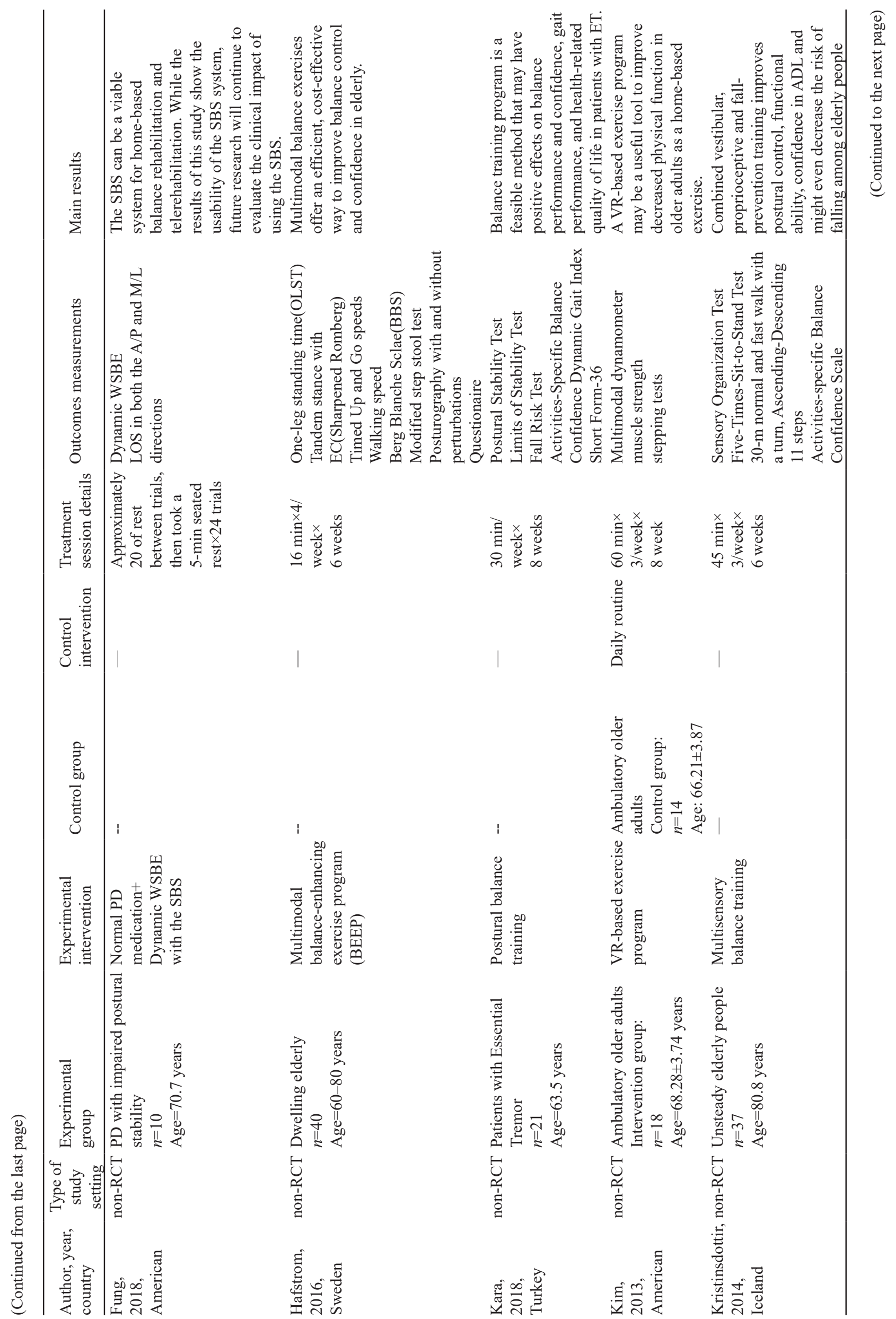




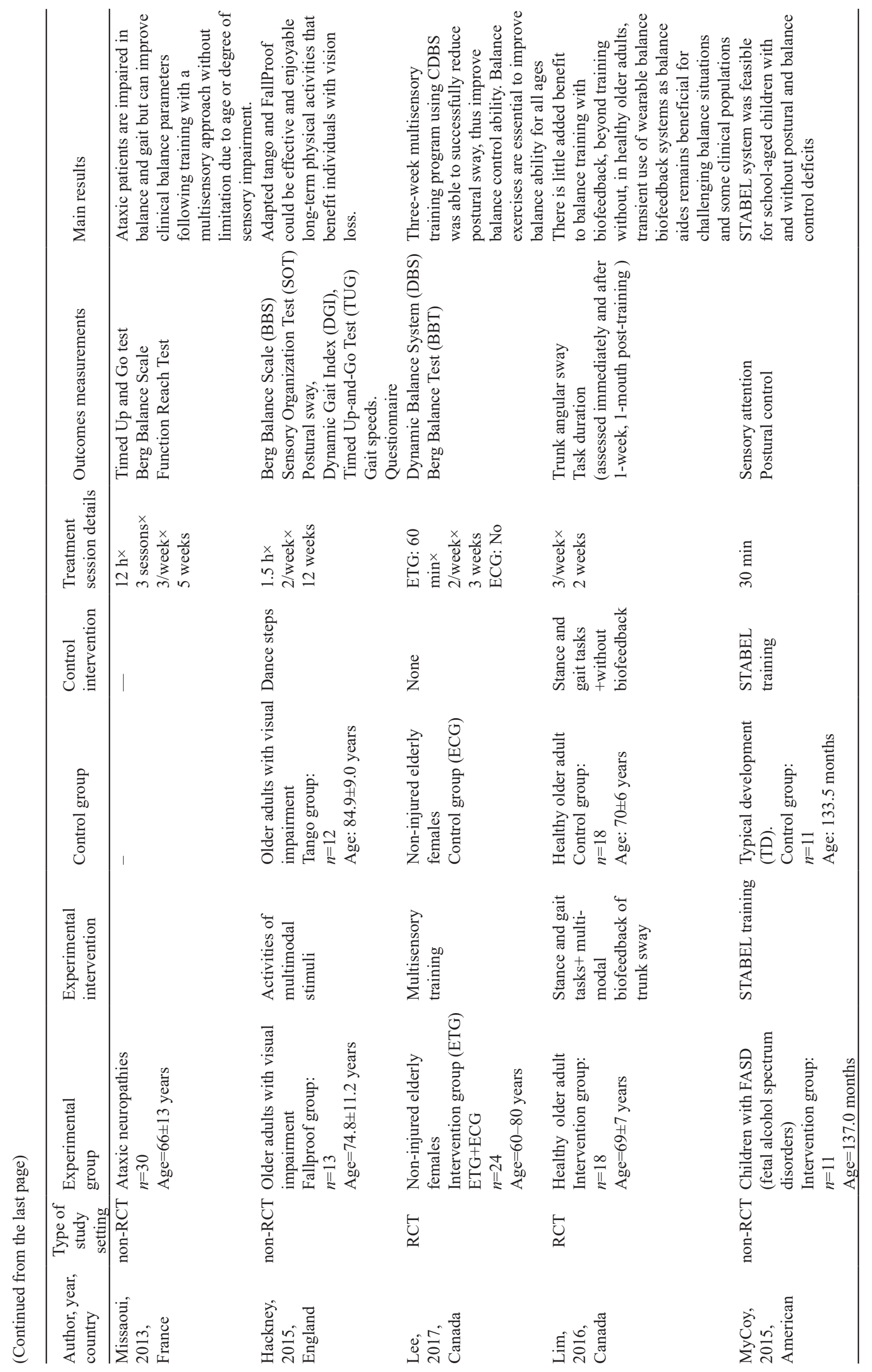

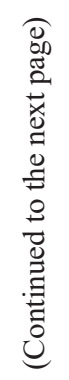




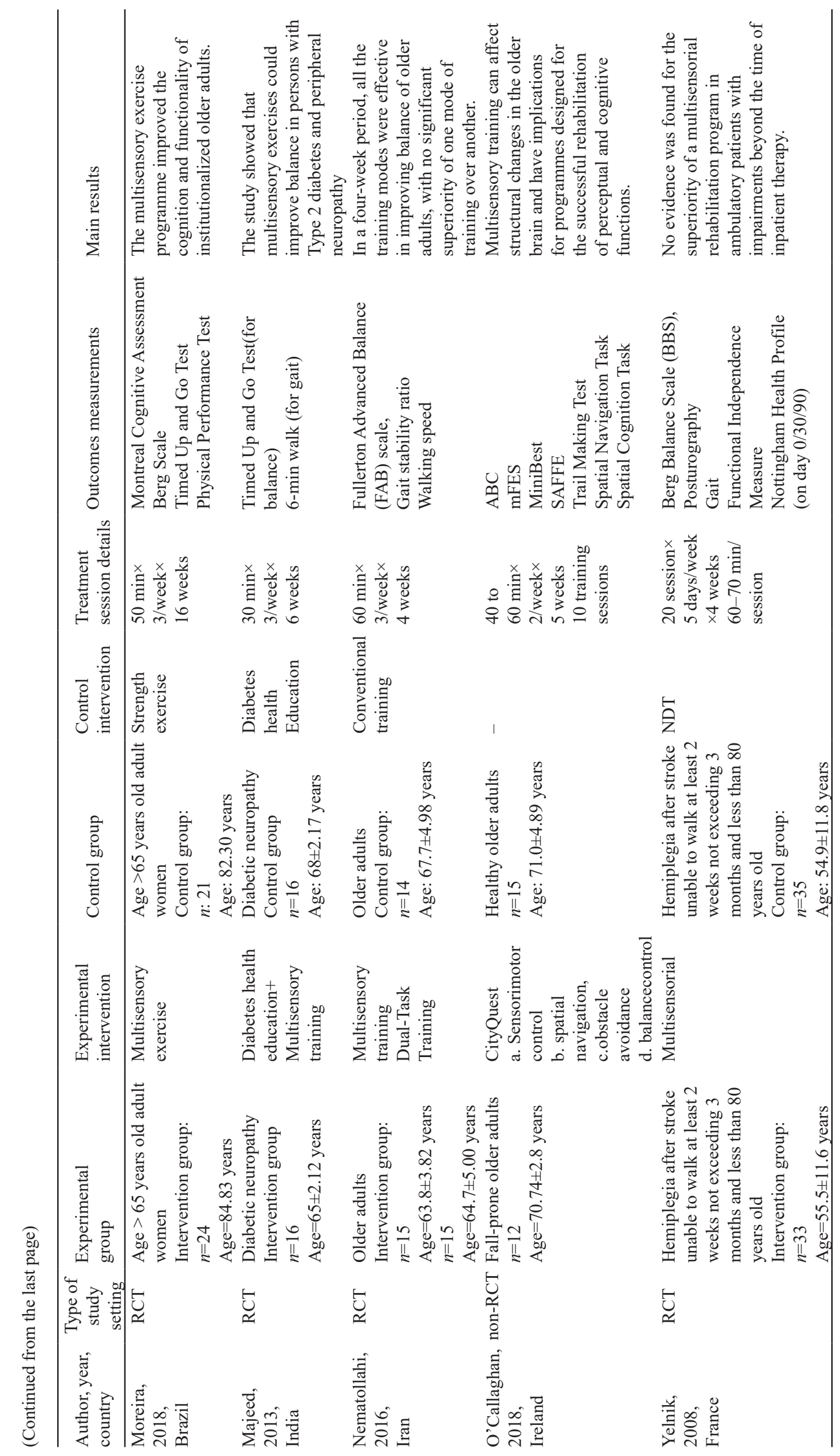




\section{DISCUSSION}

This systematic review studies evaluating multisensory exercise which improves balance in people with balance disorders (fig. 2). We identified a total of 20 moderate to high quality experimental studies representing a total of 667 participants. Most of these studies were conducted in the USA and Canada. Unfortunately, the limited number of articles retrieved and substantial heterogeneity across the retrieved studies did not enable us to conduct a meta-analysis. To our knowledge, this is the only systematic review exploring the effectiveness of multisensory exercise to improve balance in people with balance disorders.

\subsection{Interventions Evaluated by Included Studies}

The studies included in this review all but one ${ }^{[29]}$ found the superiority of multisensory exercises in patients with balance disorders. The interventions most commonly evaluated by included studies were multisensory exercises, which consist of activities simultaneously stimulating at least two sensory modalities of multiple sensory systems including visual, auditory, tactile, vestibular, somatosensory systems, and so on. Auditory stimulation mainly includes music, natural sound, voice, machine work sound, closing sound, etc ${ }^{[40,41,44]}$. Tactile was stimulated using vibratory stimulation above the perception threshold of the soles of both feet at $100 \mathrm{~Hz}$ frequency, baths in alternately cold and warm water, training by touching soft hardness, texture, or 1-2 mm vibrating items $^{[20,33,36,40,41]}$.

Multisensory integration disorder refers to the abnormality in the integration of different sensations or modalities when relevant factors of balance are significantly altered, in addition to auditory and tactile stimulation, we can use fragrance to stimulate smell and food to stimulate taste for multisensory training. Four studies simultaneously stimulated vestibular, visual, and proprioceptive feelings for multisensory exercise $^{[29,30,34,42]}$. Vestibular stimulation mainly includes head stationary; head tilt, nod, rotation, etc. Visual stimulation mainly includes closing your eyes or wearing an eye mask, fixation of eyes, gaze range from small to large static, predictable and unpredictable movement, observing the color, brightness, etc. Somatosensory stimulation mainly includes solid ground, soft support surface (foam, sponge, etc.), rocking ground, etc. It is designed to improve foot dexterity through handling, collecting objects or writing with the toes. Furthermore, we found that visual and proprioceptive stimuli were more than vestibular, auditory, and tactile stimuli in multisensory exercise. It is worth mentioning that multi-modal biofeedback systems are applied to the research of multisensory exercise, overcome the aforementioned limitations of current biofeedback systems, and develop a multi-modal head-mounted biofeedback system that provides sensory information to the wearer by using: (1) bilateral audio bone-conducted signals; (2) an array of vibrotactile signals at the head; and (3) an augmenting visual signal ${ }^{[40]}$.

\subsection{Mechanisms of Multisensory Exercises Impro- ving Balance}

From anatomical point of view, gray matter volume increased after multisensory exercises ${ }^{[35]}$. Previous studies showed that the volume of the temporal lobe, cerebellum and hippocampus decreased with ag $\mathrm{e}^{[53]}$ and the reduced gray matter volume was associated with poor balance $\mathrm{e}^{[54]}$. Multiple areas in the brain are heavily involved in multisensory information processing, such as superior colliculus ${ }^{[5]}$ and the posterior parietal cortex ${ }^{[56]}$. Our study suggested that multisensory exercises increased brain volume to enhance the ability to process multisensory information and maintain balance. Balance requires multisensory integration $(\mathrm{MSI})^{[21]}$, i.e., the process by which multisensory inputs are combined by the nervous system to form a stable and coherent percept of the world, ensuing responses of the body ${ }^{[57-59]}$. When sensory afferents from the environment are reduced, other sensory afferents will compensate for the reduction ${ }^{[60]}$. MSI is modulated by both bottom-up and top-down factors ${ }^{[61]}$. This study suggested that multisensory exercises might improve MSI by increasing bottom-up information inputs and

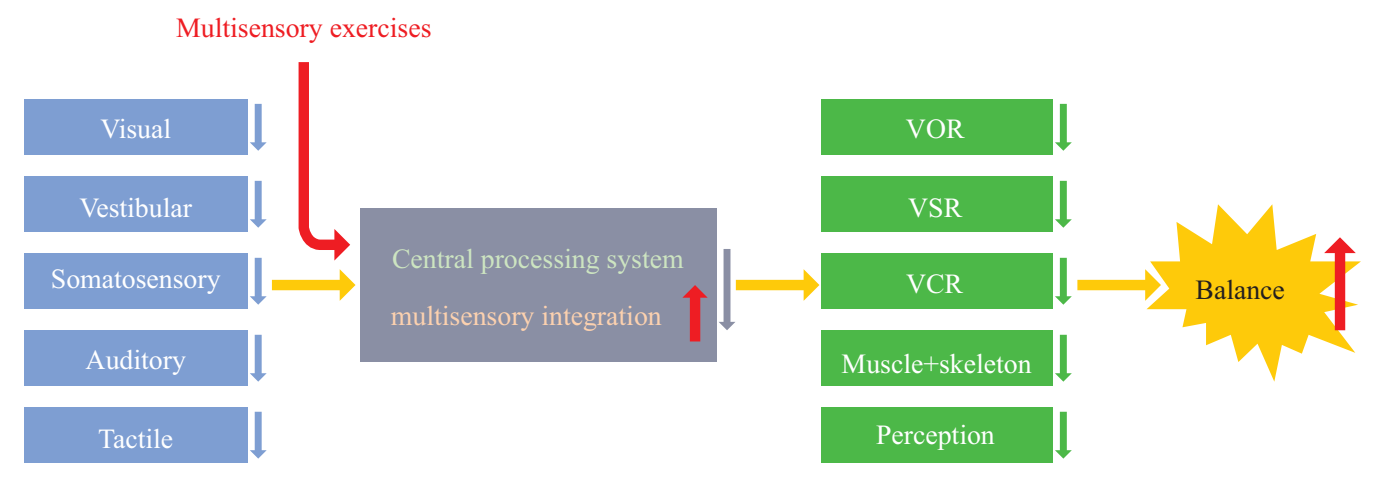

Fig. 2 Multisensory exercise improves balance in people with balance disorders

VOR: vestibular-ocular reflex; VSR: vestibulospinal reflex; VCR: vestibulocolic reflex 
Table 3 Quality assessment of eligible non-randomized studies

\begin{tabular}{|c|c|c|c|c|c|c|c|c|c|c|}
\hline Publications & Q1 & Q2 & Q3 & Q4 & Q5 & Q6 & Q7 & Q8 & Q9 & Total \\
\hline Missaoui 2013 & $\mathrm{Y}$ & $\mathrm{Y}$ & $\mathrm{Y}$ & $\mathrm{N}$ & $\mathrm{N}$ & $\mathrm{Y}$ & $\mathrm{Y}$ & $\mathrm{U}$ & $\mathrm{Y}$ & $6 / 9$ \\
\hline Allison 2018 & $\mathrm{Y}$ & $\mathrm{Y}$ & $\mathrm{Y}$ & $\mathrm{N}$ & $\mathrm{N}$ & $\mathrm{Y}$ & $\mathrm{Y}$ & $\mathrm{Y}$ & $\mathrm{Y}$ & $7 / 9$ \\
\hline Hackney 2015 & $\mathrm{Y}$ & Y & Y & $\mathrm{Y}$ & $\mathrm{N}$ & $\mathrm{Y}$ & $\mathrm{Y}$ & $\mathrm{Y}$ & $\mathrm{Y}$ & $8 / 9$ \\
\hline McCoy 2015 & $\mathrm{Y}$ & $\mathrm{Y}$ & $\mathrm{Y}$ & $\mathrm{N}$ & $\mathrm{Y}$ & $\mathrm{Y}$ & $\mathrm{Y}$ & $\mathrm{Y}$ & $\mathrm{Y}$ & $8 / 9$ \\
\hline Kristinsdottir 2014 & $\mathrm{Y}$ & $\mathrm{Y}$ & $\mathrm{Y}$ & $\mathrm{N}$ & $\mathrm{N}$ & $\mathrm{Y}$ & $\mathrm{Y}$ & $\mathrm{Y}$ & $\mathrm{Y}$ & $7 / 9$ \\
\hline O'Callaghan 2017 & $\mathrm{Y}$ & $\mathrm{Y}$ & $\mathrm{Y}$ & $\mathrm{Y}$ & $\mathrm{N}$ & $\mathrm{Y}$ & $\mathrm{Y}$ & $\mathrm{U}$ & $\mathrm{Y}$ & $7 / 9$ \\
\hline Kara 2018 & $\mathrm{Y}$ & $\mathrm{Y}$ & $\mathrm{Y}$ & $\mathrm{N}$ & $\mathrm{N}$ & Y & $\mathrm{Y}$ & $\mathrm{Y}$ & $\mathrm{Y}$ & $7 / 9$ \\
\hline Fung 2018 & $\mathrm{Y}$ & $\mathrm{Y}$ & $\mathrm{Y}$ & $\mathrm{N}$ & $\mathrm{N}$ & $\mathrm{Y}$ & $\mathrm{Y}$ & $\mathrm{Y}$ & $\mathrm{Y}$ & $7 / 9$ \\
\hline Kim 2013 & $\mathrm{Y}$ & $\mathrm{Y}$ & $\mathrm{Y}$ & $\mathrm{Y}$ & $\mathrm{Y}$ & $\mathrm{Y}$ & $\mathrm{Y}$ & $\mathrm{Y}$ & $\mathrm{Y}$ & $9 / 9$ \\
\hline Hafstrom 2016 & $\mathrm{Y}$ & $\mathrm{Y}$ & $\mathrm{Y}$ & $\mathrm{N}$ & $\mathrm{Y}$ & Y & Y & $\mathrm{Y}$ & $\mathrm{Y}$ & $8 / 9$ \\
\hline Alum 2011 & $\mathrm{Y}$ & $\mathrm{Y}$ & $\mathrm{Y}$ & $\mathrm{Y}$ & $\mathrm{Y}$ & $\mathrm{Y}$ & $\mathrm{Y}$ & $\mathrm{Y}$ & $\mathrm{Y}$ & $9 / 9$ \\
\hline
\end{tabular}

Y, yes; N, no; U, unclear

Q1: Is it clear in the study what is the "cause" and what is the "effect" (i.e. there is no confusion about which variable comes first)?

Q2: Were the participants included in any comparisons similar?

Q3: Were the participants included in any comparisons receiving similar treatment/care, other than the exposure or intervention of interest?

Q4: Was there a control group?

Q5: Were there multiple measurements of the outcome both pre and post the intervention/exposure?

Q6: Was follow up complete and if not, were differences between groups in terms of their follow up adequately described and analyzed?

Q7: Were the outcomes of participants included in any comparisons measured in the same way?

Q8: Were outcomes measured in a reliable way?

Q9: Was appropriate statistical analysis used?

Table 4 Quality assessment result of RCTs

\begin{tabular}{lcccccccccccccc}
\hline Publications & Q1 & Q2 & Q3 & Q4 & Q5 & Q6 & Q7 & Q8 & Q9 & Q10 & Q11 & Q12 & Q13 & Total \\
\hline Majeed 2013 & Y & Y & Y & Y & U & U & Y & Y & Y & Y & Y & Y & U & $10 / 13$ \\
Alfieri 2010 & U & U & Y & Y & N & N & Y & Y & Y & Y & Y & Y & Y & $10 / 13$ \\
Bellomo 2009 & U & Y & Y & Y & U & U & Y & Y & Y & Y & Y & Y & Y & $10 / 13$ \\
Lee 2017 & Y & Y & Y & Y & U & U & Y & Y & Y & Y & Y & Y & Y & $11 / 13$ \\
Lim 2016 & U & U & Y & Y & N & N & Y & Y & Y & Y & Y & Y & Y & $9 / 13$ \\
Moreira 2018 & Y & Y & Y & Y & Y & Y & Y & Y & Y & Y & Y & Y & Y & $13 / 13$ \\
Yelnik 2008 & Y & Y & Y & Y & N & N & Y & Y & Y & Y & Y & Y & Y & $11 / 13$ \\
Davis 2010 & Y & Y & U & U & U & Y & Y & Y & Y & Y & Y & Y & Y & $10 / 13$ \\
Nematollahi 2016 & Y & Y & Y & Y & Y & Y & Y & N & Y & Y & Y & Y & Y & $11 / 13$ \\
\hline Y, Ne; N & & & & & & & & & & & & & &
\end{tabular}

Y, yes; N, no; U, unclear

Q1: Was true randomization used for assignment of participants to treatment groups?

Q2: Was allocation to treatment groups concealed?

Q3: Were treatment groups similar at the baseline?

Q4: Were participants blind to treatment assignment?

Q5: Were those delivering treatment blind to treatment assignment?

Q6: Were outcomes assessors blind to treatment assignment?

Q7: Were treatment groups treated identically other than the intervention of interest?

Q8: Was follow up complete and if not, were differences between groups in terms of their follow up adequately described and analyzed? Q9: Were participants analyzed in the groups to which they were randomized?

Q10: Were outcomes measured in the same way for treatment groups?

Q11: Were outcomes measured in a reliable way?

Q12: Was appropriate statistical analysis used?

Q13: Was the trial design appropriate, and any deviations from the standard RCT design (individual randomization, parallel groups) accounted for in the conduct and analysis of the trial?

modulating top-down signals. Multisensory exercises stimulate multiple sensory inputs of various sources simultaneously to increase bottom-up information inputs $^{[13,61]}$. If information of an object requires integration of more than one sensory systems, it captures the attention of an information receiver more efficiently, and proves that bottom-up integration can "drive" attention ${ }^{[62]}$. On the other hand, the bottom- 
up information inputs can be modulated by the topdown signals of the brain ${ }^{[63]}$. The mammalian brain is constantly learning from task-relevant sensory stimuli in the environment ${ }^{[61]}$ which changes the way of sensory cortex responding to the task-relevant sensory of stimulation ${ }^{[64]}$. Learning-enhanced sensory responses are further facilitated by attention and task engagement ${ }^{[65,66]}$.

Selective attention to important features of taskrelevant stimuli can also enhance sensory responses ${ }^{[67]}$. When multiple sensory systems are repeatedly stimulated by multisensory exercises, the brain might also learn from relevant sensory stimuli, put more attention on exercises-relevant sensory stimuli and modulate bottom-up inputs to improve balance.

Another possible mechanism is that multisensory exercises may enhance receptors' sensitivity of different sensory systems ${ }^{[13]}$. Additionally, the ability for unisensory system processing might also get improved after multisensory exercises, since declined unisensory processing ability might impair balance performance ${ }^{[68,69]}$.

\subsection{Synthesized Findings}

Analysis in terms of study type showed that, in both RCTs and non-randomized studies, multisensory exercises could effectively improve balance. Analysis in terms of intervention time showed that multisensory exercises worked within 6 weeks. Our analysis indicated that the optimal intervention time might be less than 6 weeks and multisensory exercises beyond 6 weeks are not necessary. And animal studies showed the time taken for vestibular compensation was no more than 7 days ${ }^{[70,71]}$. The intervention time should suffice to modulate central nervous plasticity. New neural networks may be activated and homeostasis may be achieved in the CNS. Moreover, the effect of multisensory exercises on balance might linger for a long time after the exercises ceased ${ }^{[34,37]}$. A follow-up study by Hackney et al. exhibited that the changes in SOT and BBS remained from the end of the intervention to one-month after the termination ${ }^{[37]}$. And Kritinsdottir et al ${ }^{[34]}$ found that the episodes of fall dropped during a time of 6-month. The long-term effect may be attributed to changes in the CNS effected by multisensory exercises. Multisensory exercises may elicit neurogenesis and synapse formation in the brain and new connections may develop between different brain regions. Besides, analysis showed no significant difference in balance improvement among patients with various etiologies. The result suggested that multisensory exercises are equally effective for patients with both central or peripheral nervous system disorders.

Multisensory exercises out-performed physical exercises in balance improvemet ${ }^{[36]}$. Alfieri et al reported that after 3 months of training, multisensory exercises decreased the time to complete TUG by $11.85 \%$, while strength exercises shortened the time by only $3 \%$, and the difference between the groups was statistically significant ${ }^{[36]}$. The results suggested that multisensory exercises work better on dynamic balance than strength exercises, possibly because multisensory exercises could enhance multisensorial receptors' sensitivity ${ }^{[72,}{ }^{73]}$. On the other hand, the strength exercises mainly target muscle groups. Strength training could significantly slow movement of pressure center while multisensory exercises could shorten the distance over which pressure center moved ${ }^{[36]}$. This suggested that multi-sensorial stimulation could help subjects develop optimal strategies for postural control, thereby minimizing body sway ${ }^{[74]}$.

Interestingly, we found that participants' mean age of the included studies were over 60 years old. As we know it, the incidence of balance disorders in older adults is higher than in young people. And aging progressively impairs sight, vestibular input, and somatosensory information, and reduces the number of muscle and nerve fibers, resulting in an impaired environmental perception and muscle strength ${ }^{[1,59,75]}$. Besides, impaired multisensory integration makes the elderly people more vulnerable to fall ${ }^{[76]}$. Patients with vestibular dysfunction were not included in our analysis, since they received vestibular rehabilitation, instead of multisensory exercises, as the major therapy.

\subsection{Strengths and Limitations}

The present systematic review was performed according to the PRISMA statement ${ }^{[26]}$. This study had some limitations. We conducted analysis in terms of study type, intervention time and the underlying etiology of balance disorders, but gave up meta-analysis due to limited number of articles retrieved and substantial heterogeneity. Despite the comprehensiveness of our search, there is always a possibility that in a systematic review, we may have failed to uncover all available literature. Readers should also be aware that we have only included the strongest available evidence based on our critical appraisal of existing studies. Due to limited information about diseases of participants, we didn't conduct analysis on the basis of disease severity. Readers are further cautioned that the findings of studies included in this review reflect the effectiveness of outcome measures for balance and caution should be taken when attempting to generalize to those of other factors

To sum up, the findings of studies included in this review combined with knowledge of existing literature suggest that multisensory exercises may be a promising avenue for future research in terms of developing and evaluating interventions that target multisensory integration in balance disorder. We confirmed that the multisensory exercises were effective in improving balance in people with balance disorders. Multisensory 
exercises could lower the risk of fall and enhance confidence level so as to improve the quality of life. Our results provided a new basis for the design of future training programs aimed to improve balance. Further research is needed to investigate the optimal strategy of multisensory exercises and explore the underlying neural and molecular mechanisms of balance improvement brought by multisensory exercises.

\section{Open Access}

This article is licensed under a Creative Commons Attribution 4.0 International License https://creativecommons.org/licenses/by/4.0/), which permits use, sharing, adaptation, distribution and reproduction in any medium or format, as long as you give appropriate credit to the original author(s) and the source, provide a link to the Creative Commons licence, and indicate if changes were made. The images or other third party material in this article are included in the article's Creative Commons licence, unless indicated otherwise in a credit line to the material. If material is not included in the article's Creative Commons licence and your intended use is not permitted by statutory regulation or exceeds the permitted use, you will need to obtain permission directly from the copyright holder. To view a copy of this licence, visit http://creativecommons. org/licenses/by/4.0/.

\section{Conflict of Interest Statement}

All authors declare no conflicts of interest.

Author Su-ling ZHANG is a young member of the Editorial Board for Current Medical Science. The paper was handled by the other editor and has undergone rigrous peer review process. Author Su-ling ZHANG was not involved in the journal's review of, or decisions related to, this manuscript.

\section{REFERENCES}

1 Alexander NB. Postural control in older adults. J Am Geriatr Soc, 1994,42(1):93-108

2 Woollacott $\mathrm{MH}$, Tang PF. Balance control during walking in the older adult: research and its implications. Phys Ther, 1997,77(6):646-660

3 Goldie PA, Bach TM, Evans OM. Force platform measures for evaluating postural control: reliability and validity. Arch Phys Med Rehabil, 1989,70(7):510-517

4 Distefano LJ, Clark MA, Padua DA. Evidence supporting balance training in healthy individuals: a systemic review. J Strength Cond Res, 2009,23(9):27182731

5 Yim-Chiplis PK, Talbot LA. Defining and measuring balance in adults. Biol Res Nurs, 2000,1(4):321-331

6 Jh J. In: Taylor J, Holmes G, Walshe FMR, eds. Selected writings of John Hughlings Jackson. London: Hodder and Stoughton. 1932,2.

7 Nutt JG, Marsden CD, Thompson PD. Human walking and higher-level gait disorders, particularly in the elderly. Neurology, 1993,43(2):268-279

8 Berg K. Balance and its measure in the elderly: A review. Physiotherapy Canada, 1989,41(5):240-246

9 Rossat A, Fantino B, Nitenberg C, et al. Risk factors for falling in community-dwelling older adults: which of them are associated with the recurrence of falls? J Nutr Health Aging, 2010,14(9):787-791

10 WHO (2018) Falls. WHO Fact Sheets. https://www. who.int/en/news-room/fact-sheets/detail/falls

11 Pellicer MG, Lusar AC, Casanovas JM, et al. Effectiveness of a multimodal exercise rehabilitation program on walking capacity and functionality after a stroke. J Exerc Rehabil, 2017,13(6):666-675

12 Cohen HS, Kimball KT. Decreased ataxia and improved balance after vestibular rehabilitation. Otolaryngol Head Neck Surg, 2004,130(4):418-425

$13 \mathrm{Hu} \mathrm{MH}$, Woollacott MH. Multisensory training of standing balance in older adults: I. Postural stability and one-leg stance balance. J Gerontol, 1994,49(2):M52-61

14 Costa PB, Graves BS, Whitehurst M, et al. The acute effects of different durations of static stretching on dynamic balance performance. J Strength Cond Res, 2009,23(1):141-147

15 American College of Sports M. American College of Sports Medicine position stand. Progression models in resistance training for healthy adults. Med Sci Sports Exerc, 2009,41(3):687-708

16 Lam FM, Huang MZ, Liao LR, et al. Physical exercise improves strength, balance, mobility, and endurance in people with cognitive impairment and dementia: a systematic review. J Physiother, 2018,64(1):4-15

17 Whitney SL, Alghwiri AA, Alghadir A. An overview of vestibular rehabilitation. Handb Clin Neurol, 2016,137: 187-205

18 Hall CD, Cox LC. The role of vestibular rehabilitation in the balance disorder patient. Otolaryngol Clin North Am, 2009,42(1):161-169

19 Cruz J, Marques A, Barbosa AL, et al. Effects of a motor and multisensory-based approach on residents with moderate-to-severe dementia. Am J Alzheimers Dis Other Demen, 2011,26(4):282-289

20 Allison LK, Kiemel T, Jeka JJ. Sensory-Challenge Balance Exercises Improve Multisensory Reweighting in Fall-Prone Older Adults. J Neurol Phys Ther, 2018, 42(2):84-93

21 Stein BE, Meredith MA. Multisensory integration. Neural and behavioral solutions for dealing with stimuli from different sensory modalities. Ann N Y Acad Sci, 1990,608:51-65; discussion -70

22 Barry E. Stein BaR. Neural development of multisensory integration. Multisensory Perception, 2020:57-87

23 Tjernström F, Zur O, Jahn K. Current concepts and future approaches to vestibular rehabilitation. J Neurol, 2016,263 Suppl 1:S65-70

24 Lee AC, Magee DJ. Multisensory Training for Postural Sway Control in Non-Injured Elderly Females. J Fundam Appl Sci, 2017,9:1116-1131

25 Bayouk JF, Boucher JP, Leroux A. Balance training following stroke: effects of task-oriented exercises with and without altered sensory input. Int J Rehabil Res, 2006,29(1):51-59

26 Moher D, Liberati A, Tetzlaff J, et al. Preferred reporting items for systematic reviews and meta-analyses: the PRISMA statement. PLoS Med, 2009,6(7):e1000097

27 Tufanaru CMZ, Aromataris E, Campbell J, et al. In: Aromataris E, Munn Z, eds. Chapter 3: Systematic reviews of effectiveness. Australia: 2020. Available 
from https://synthesismanual.jbi.global

28 Fung A, Lai EC, Lee BC. Usability and Validation of the Smarter Balance System: An Unsupervised Dynamic Balance Exercises System for Individuals With Parkinson's Disease. IEEE Trans Neural Syst Rehabil Eng, 2018,26(4):798-806

29 Yelnik AP, Le Breton F, Colle FM, et al. Rehabilitation of balance after stroke with multisensorial training: a single-blind randomized controlled study. Neurorehabil Neural Repair, 2008,22(5):468-476

30 Mccoy SW, Jirikowic T, Price R, et al. Virtual Sensorimotor Balance Training for Children With Fetal Alcohol Spectrum Disorders: Feasibility Study. Phys Ther, 2015,95(11):1569-1581

31 Moreira NB, Gonçalves G, Da Silva $\mathrm{T}$, et al. Multisensory exercise programme improves cognition and functionality in institutionalized older adults: A randomized control trial. Physiother Res Int, 2018,23(2): e1708

32 Ai CL, Sciences A. Multisensory training for postural sway control in non-injured elderly females. J Fundam Appl Sci, 2017,9(6S):1116-1131

33 Missaoui B, Thoumie P. Balance training in ataxic neuropathies. Effects on balance and gait parameters. Gait Posture, 2013,38(3):471-476

34 Kristinsdottir EK, Baldursdottir B. Effect of multisensory balance training for unsteady elderly people: pilot study of the "Reykjavik model". Disabil Rehabil, 2014,36(14):1211-1218

35 O'callaghan G, O'dowd A, Stapleton J, et al. Changes in Regional Brain Grey-Matter Volume Following Successful Completion of a Sensori-Motor Intervention Targeted at Healthy and Fall-Prone Older Adults. Multisens Res, 2018,31(3-4):317-344

36 Alfieri FM, Riberto M, Gatz LS, et al. Functional mobility and balance in community-dwelling elderly submitted to multisensory versus strength exercises. Clin Interv Aging, 2010,5:181-185

37 Hackney ME, Hall CD, Echt KV, et al. Multimodal Exercise Benefits Mobility in Older Adults With Visual Impairment: A Preliminary Study. J Aging Phys Act, 2015,23(4):630-639

38 Kutty NAM, Majida NJDC. Effects of Multisensory Training on Balance and Gait in Persons with Type 2 Diabetes: A Randomised Controlled Trial. Disab CBR Inclus Develop, 2013,24(2):79-97

39 Kara B, Kahraman T, Tomruk MS, et al. The Effects of a Postural Balance Training Program on Balance, Gait and Health-Related Quality of Life in Patients with Essential Tremor. Turk Noroloji Dergisi, 2018,2018(1):43-48

40 Davis JR, Carpenter MG, Tschanz R, et al. Trunk sway reductions in young and older adults using multi-modal biofeedback. Gait Posture, 2010,31(4):465-472

41 Allum JH, Carpenter MG, Horslen BC, et al. Improving impaired balance function: real-time versus carry-over effects of prosthetic feedback. Annu Int Conf IEEE Eng Med Biol Soc, 2011,2011:1314-1318

42 Hafström A, Malmström EM, Terdèn J, et al. Improved Balance Confidence and Stability for Elderly After 6 Weeks of a Multimodal Self-Administered BalanceEnhancing Exercise Program: A Randomized Single Arm Crossover Study. Gerontol Geriatr Med, 2016,2:

\section{9}

43 Kim J, Son J, Ko N, et al. Unsupervised virtual realitybased exercise program improves hip muscle strength and balance control in older adults: a pilot study. Arch Phys Med Rehabil, 2013,94(5):937-943

44 Bellomo RG, Iodice P, Savoia V, et al. Balance and posture in the elderly: an analysis of a sensorimotor rehabilitation protocol. Int J Immunopathol Pharmacol, 2009,22(3 Suppl):37-44

45 Lim SB, Horslen BC, Davis JR, et al. Benefits of multisession balance and gait training with multi-modal biofeedback in healthy older adults. Gait Posture, 2016, 47:10-17

46 Nematollahi A, Kamali F, Ghanbari A, et al. Improving Balance in Older People: A Double-Blind Randomized Clinical Trial of Three Modes of Balance Training. J Aging Phys Act, 2016,24(2):189-195

47 Nashner LM, Peters JF. Dynamic posturography in the diagnosis and management of dizziness and balance disorders. Neurol Clin, 1990,8(2):331-349

48 Podsiadlo D, Richardson S. The timed "Up \& Go": a test of basic functional mobility for frail elderly persons. J Am Geriatr Soc. 1991,32:142-148

49 Berg K, Wood-Dauphinee S, Williams JI, et al. Measuring balance in the elderly: preliminary development of an instrument. Physiotherapy Canada, 1989,41(2):304-311

50 Yelnik A, Bonan I. Clinical tools for assessing balance disorders. Neurophysiol Clin, 2008,38(6):439-445

51 Powell LE, Myers AM. The Activities-specific Balance Confidence (ABC) Scale. J Gerontol A Biol Sci Med Sci, 1995,50a(1):M28-34

52 Arkadir D, Louis ED. The balance and gait disorder of essential tremor: what does this mean for patients? Ther Adv Neurol Disord, 2013,6(4):229-236

53 Peters R. Ageing and the brain. Postgrad Med J, 2006,82(964):84-88

54 Rosano C, Aizenstein H, Brach J, et al. Special article: gait measures indicate underlying focal gray matter atrophy in the brain of older adults. J Gerontol A Biol Sci Med Sci, 2008,63(12):1380-1388

55 Stein Be MM. The Merging of the Senses. Cambridge, MA: MIT Press, 1993.

56 Ghazanfar AA, Schroeder CE. Is neocortex essentially multisensory? Trends Cogn Sci, 2006,10(6):278-285

57 Jenkins HA, Furman JM, Gulya AJ, et al. Dysequilibrium of aging. Otolaryngol Head Neck Surg, 1989,100(4): 272-282

58 Katsarkas A. Dizziness in aging: a retrospective study of 1194 cases. Otolaryngol Head Neck Surg, 1994,110(3): 296-301

59 Woollacott M, Shumway-Cook A. Attention and the control of posture and gait: a review of an emerging area of research. Gait Posture, 2002,16(1):1-14

60 Horak FB, Nashner LM, Diener HC. Postural strategies associated with somatosensory and vestibular loss. Exp Brain Res, 1990,82(1):167-177

61 Choi I, Lee JY, Lee SH. Bottom-up and top-down modulation of multisensory integration. Curr Opin Neurobiol, 2018, 52:115-122

62 Talsma D. Predictive coding and multisensory integration: an attentional account of the multisensory mind. Front Integr Neurosci, 2015,9:19 
63 Talsma D, Senkowski D, Soto-Faraco S, et al. The multifaceted interplay between attention and multisensory integration. Trends Cogn Sci, 2010,14(9): 400-410

64 Le Merre P, Esmaeili V, Charriere E, et al. RewardBased Learning Drives Rapid Sensory Signals in Medial Prefrontal Cortex and Dorsal Hippocampus Necessary for Goal-Directed Behavior. Neuron, 2018,97(1):83-91 e5

65 Caras ML, Sanes DH. Top-down modulation of sensory cortex gates perceptual learning. Proc Natl Acad Sci USA, 2017,114(37):9972-9977

66 Li W, Piech V, Gilbert CD. Perceptual learning and topdown influences in primary visual cortex. Nat Neurosci, 2004,7(6):651-657

67 Reynolds JH, Chelazzi L. Attentional modulation of visual processing. Annu Rev Neurosci, 2004,27:611647

68 Anson E, Jeka J. Perspectives on Aging Vestibular Function. Front Neurol, 2015,6:269

69 Lord SR. Visual risk factors for falls in older people. Age Ageing, 2006,35 Suppl 2:ii42-ii5

70 Zhou L, Zhou W, Zhang S, et al. BDNF signaling in the rat cerebello-vestibular pathway during vestibular compensation: BDNF signaling in vestibular compensation. FEBS J, 2015,282(18):3579-3591

71 Zhou W, Zhou LQ, Shi H, et al. Expression of glycine receptors and gephyrin in rat medial vestibular nuclei and flocculi following unilateral labyrinthectomy. Int J Mol Med, 2016,38(5):1481-1489

72 Rogers ME, Rogers NL, Takeshima N, et al. Methods to assess and improve the physical parameters associated with fall risk in older adults. Prev Med, 2003,36(3):255264

73 Bean JF, Vora A, Frontera WR. Benefits of exercise for community-dwelling older adults. Arch Phys Med Rehabil, 2004,85(7 Suppl 3):S31-42; quiz S3-4

74 Nagy E, Feher-Kiss A, Barnai M, et al. Postural control in elderly subjects participating in balance training. Eur J Appl Physiol, 2007,100(1):97-104

75 Shaffer SW, Harrison AL. Aging of the somatosensory system: a translational perspective. Phys Ther, 2007, 87(2):193-207

76 Zhang SL, Zhu YT, Tian E, et al. Impaired Multisensory Integration Predisposes the Elderly People to Fall: A Systematic Review. Front Neurosci, 2020,14:411

(Received Jun. 30, 2021; accepted Aug. 4, 2021) 